\title{
The Eleven Antenna: A Compact Low-Profile Decade Bandwidth Dual Polarized Feed for Reflector Antennas
}

\author{
Rikard Olsson, Student Member, IEEE, Per-Simon Kildal, Fellow, IEEE, and Sander Weinreb, Life Fellow, IEEE
}

\begin{abstract}
A novel dual polarized ultrawide-band (UWB) feed with a decade bandwidth is presented for use in both single and dual reflector antennas. The feed has nearly constant beam width and $11 \mathrm{dBi}$ directivity over at least a decade bandwidth. The feed gives an aperture efficiency of the reflector of $66 \%$ or better over a decade bandwidth when the subtended angle toward the sub or main reflector is about $53^{\circ}$, and an overall efficiency better than $47 \%$ including mismatch. The return loss is better than $5 \mathrm{~dB}$ over a decade bandwidth. The calculated results have been verified with measurements on a linearly polarized lab model. The feed has no balun as it is intended to be integrated with an active $180^{\circ}$ balun and receiver. The feed is referred to as the Eleven antenna because its basic configuration is two parallel dipoles 0.5 wavelengths apart and because it can be used over more than a decade bandwidth with $11 \mathrm{dBi}$ directivity. We also believe that $11 \mathrm{~dB}$ return loss is achievable in the near future.
\end{abstract}

Index Terms-Log-periodic antenna, multifunction, reflector antenna, reflector antenna feed, square kilometer array (SKA), ultrawide-band (UWB).

\section{INTRODUCTION}

$\mathbf{R}$ ECENTLY, there has been a growing interest in reducing the number of antennas on different platforms by combining several functions in the same antenna. To achieve this, the multifunction antenna needs to be either very broadband or multiband to cover the frequencies of all functions. There is also an increasing interest in antennas with extremely large bandwidth in connection with proposed scientific systems such as e.g., the so-called square kilometer array (SKA) radio telescope covering frequencies from $100 \mathrm{MHz}$ to $25 \mathrm{GHz}$. The term ultrawide-band (UWB) is often used in connection with new broadband technology. The present paper deals with the development of a decade bandwidth feed for the dual reflector antennas proposed to be used for SKA in the US proposal.

Reflector antennas have existed since the beginning of wireless communication. Heinrich Hertz used a cylindrical reflector with dipole feed when he experimentally discovered electromagnetic waves in 1888 . When the radar was developed during the second world war, the most common feeds were resonant

\footnotetext{
Manuscript received February 15, 2005; revised July 28, 2005. This work was supported by the California Institute of Technology and Cornell University under Contract with ASTRON in connection with the U.S. SKA proposal.

R. Olsson and P.-S. Kildal are with the Antenna group at Chalmers University of Technology, Goteborg, S-412 96, Sweden (e-mail: rikard.olsson@elmagn.chalmers.se; simon@elmagn.chalmers.se).

$\mathrm{S}$. Weinreb is with the Department of Electrical Engineering, California Institute of Technology, Pasadena, CA 91125 USA (e-mail: sweinreb@ caltech.edu). Digital Object Identifier 10.1109/TAP.2005.863392
}

halfwave dipoles and small open-ended waveguides or horn antennas [1], [2]. The halfwave dipole got improved efficiency by locating it on a circular disk, but still the radiation pattern was very different in E- and H-planes. This caused low efficiency, and high spillover and cross polarization; the latter being detrimental in dual polarized systems. There exist two easy ways of compensating for these deficiencies, either by using two parallel dipoles with half wavelength spacing [3], or by locating a metal ring of about one wavelength diameter above the dipole [4], [5]. The feed described in the present paper is a log-periodic version of the dual dipole feed in [3].

The horn feeds became very popular during the eighties. In particular, corrugated horns were used to produce very good performance in terms of symmetry of the main lobe and cross polarization. They were and still are popular as feeds for dual reflector antennas, and they can have octave bandwidth [6], [7]. Corrugated horns are expensive and heavy, so simpler dielectric alternatives have been proposed [8]. Dielectric loading can also enable larger bandwidth [9], [10]. The quad-ridge horn is even more broadband, but unfortunately the efficiency and cross polarization is poor [11], [12]. A major problem with all the above mentioned horns in wide band applications is that the beam width varies strongly with frequency, unless the horn is made very large. The first compact horn feed with constant beam width and low cross polarization over almost an octave band width was published in [13]. There exist also self-supported feeds for prime-focus reflectors, mounted to the center of the main reflector with an axial tube containing the feed waveguide or cables. Such feeds are, e.g., the Clavin [14] feed and the hat feed mentioned in [15], but their bandwidths are typically $10 \%$ or smaller.

The feed in the present paper is in comparison to all of the above mentioned feeds very light weight and compact. The beamwidth is constant, and the cross-polar sidelobes are reasonably low over a decade bandwidth. It can be used for both single and dual reflector antennas, although the large beam width makes it better suited for prime-focus reflectors.

The square kilometer array (SKA) is an international project that will be the next big step in radio astronomy, if fully funded. As the name indicates the radio telescope will have a staggering total aperture area of about 1 square kilometer. This huge radio telescope will provide two orders of magnitude increase in sensitivity compared to existing radio telescopes. In order to achieve such a large aperture an array of antennas with smaller aperture has to be used. There are many different suggestions for antennas, ranging from a few tens of very large single 

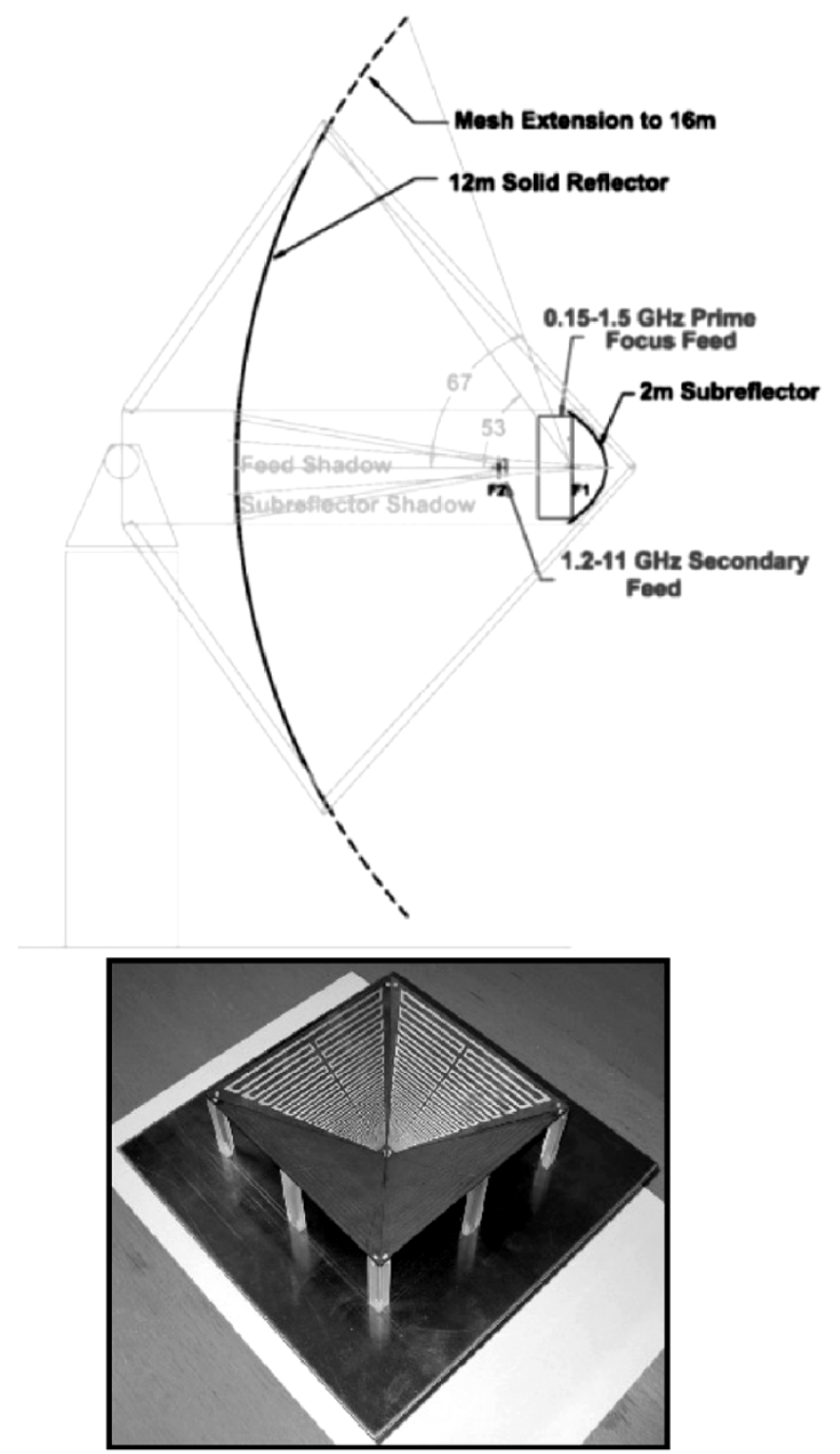

Fig. 1. Drawing of the SKA $12 \mathrm{~m} / 16 \mathrm{~m}$ symmetric antenna concept having a $12-\mathrm{m}$ parabolic (focal length is $6 \mathrm{~m}$ ) main reflector with a mesh extension to 16 $\mathrm{m}$ and a 2-m diameter subreflector, and photograph of dual polarized laboratory model of the proposed feed described in this paper. A patent is pending [23].

reflectors to large arrays of Vivaldi antennas and Luneberg lenses. The US proposal makes use of an array of around 5000 symmetric parabolic reflectors with $16 \mathrm{~m}$ diameter. In Fig. 1 a sketch of the proposed symmetric reflector of US-SKA is shown as well as a photograph of a laboratory model of the proposed feed described in the present paper. Note that the outer $4 \mathrm{~m}$ circumferential part of the reflector is a wire mesh. One of the advantages with the US proposal is that it makes use of reflector antennas that represent a well known technology which has been used in radio astronomy during several decades. The system is very wide band; the goal is to cover $100 \mathrm{MHz}$ to $25 \mathrm{GHz}$. There are many benefits from this bandwidth. The main advantage is that the same radio telescope may be used to perform several functions and thus support many different kinds of research. Key science projects for SKA include studies of the evolution of galaxies and cosmic large structures, studies on the first black holes and stars, strong field tests of gravity, the search for Earth like planets and much more [16]. The number of antennas and the bandwidth of the system have triggered a need for development of cheap ultrawide-band components. One of the more challenging components is the feed.

End-fire style log-periodic dipole arrays have since they were invented [17] been used in ultra wide band antenna design to achieve medium gain antennas for use in both antenna and electromagnetic compatability (EMC) measurements. Log-periodic antennas are not common as reflector feeds because their phase centers vary strongly with frequency [18], [19]. The phase center variation can be reduced to acceptable levels, such as e.g., in the log-periodic feed for the Allen Telescope Array (ATA) [20]. This is a radio telescope currently under construction which uses many of the same ideas as the U.S. SKA proposal, but for an array of 350 reflectors of $6 \mathrm{~m}$ diameter. Still, the ATA feed is large and there are significant losses due to phase center variation [21], so better feeds are desirable. The purpose of the present paper is to describe a compact feed with almost no phase center variation and almost constant beam width over a decade bandwidth. The described feed contains no balun as it is intended to be integrated with an active $180^{\circ}$ balun and receiver. An important advantage of the described feed is that due to its compact size it facilities cryogenic cooling. The measurements reported in the present paper have been done with a broadband commercial $180^{\circ}$ hybrid power divider replacing the balun. Some initial work on the feed was reported briefly in [22], and a patent is pending [23]. The feed has been named the Eleven antenna because its basic configuration is two parallel dipoles 0.5 wavelengths apart and because it can be used over more than a decade bandwidth with 11-dBi directivity. We also believe that $11-\mathrm{dB}$ return loss is achievable in the near future.

\section{Characterization OF FEEDS}

The antenna to be presented has been designed to feed a reflector, more particularly the reflector depicted in Fig. 1. Therefore, it is important to characterize its performance in the reflector system. This is done by the aperture efficiency in the reflector and its subefficiencies, and by spillover, input reflection coefficient, and figure of merit, as explained later.

\section{A. Subefficiencies}

The realized gain $G_{0}$ and directivity $D_{0}$ of a reflector are related by

$$
G_{0}=e_{\mathrm{ant}} D_{0} ; \quad e_{\mathrm{ant}}=e_{\mathrm{rad}} e_{\mathrm{ap}}
$$

where $e_{\mathrm{ant}}$ is the antenna efficiency, $e_{\mathrm{ap}}$ is the aperture efficiency and

$$
e_{\text {rad }}=e_{\text {reff }} e_{\text {diss }} ; \quad e_{\text {refl }}=1-\left|r^{2}\right|
$$

is the total radiation efficiency. $e_{\text {reff }}$ accounts for the input reflection coefficient $r$, i.e., impedance mismatch, and $e_{\text {diss }}$ for the dissipation losses. When we calculate the reflection coefficient $r$ we will assume $50 \Omega$ input feed line impedance. 


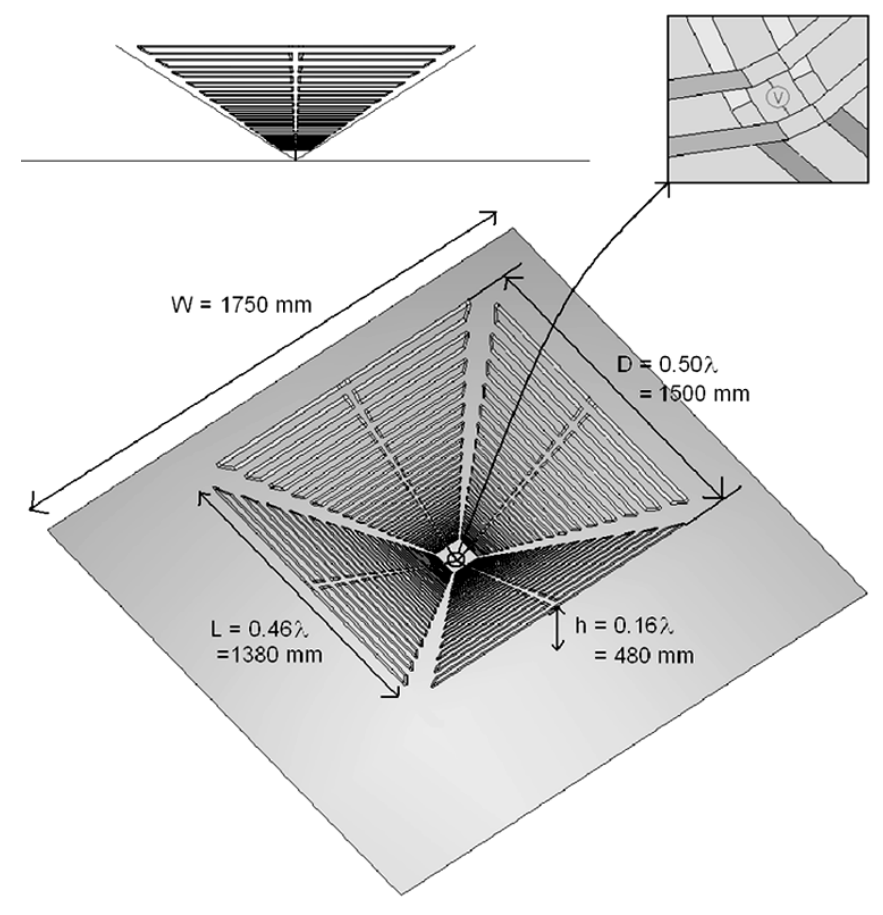

Fig. 2. Illustration of a 0.15 to $1.5 \mathrm{GHz}$ dual polarized model of the feed with key dimensions indicated. The largest value of each dimension is given in $\mathrm{mm}$. [26]. These sizes are also given in terms of the wavelength at $100 \mathrm{MHz}$. The lowest frequency of operation is $150 \mathrm{MHz}$. A side view is shown on the top left, and on the top right a more detailed view of the central part of the feed is shown.

The aperture efficiency $e_{\mathrm{ap}}$ is a measure of how efficiently the feed illuminates the reflector aperture, and it becomes unity for a uniform aperture illumination with constant phase and no spillover. The aperture efficiency can for classical symmetric reflector shapes like the SKA reflector in Fig. 1 be expressed in closed form in terms of the radiation field function of the feed. To characterize different properties of the feed we will separate the aperture efficiency in different subefficiencies, see ([24, Sec. 8.4]) and [25]. According to

$$
e_{\mathrm{ap}}=e_{\mathrm{BOR}} e_{\mathrm{sp}} e_{\mathrm{pol}} e_{\mathrm{ill}} e_{\phi}
$$

where each of the subefficiencies are explained below. The body of revolution 1 (BOR) efficiency $e_{\mathrm{BOR}}$ measures how closely the far-field resembles that of a $\mathrm{BOR}_{1}$ antenna ([24, Sec. 2.4]) and [26]. The far-field of a $\mathrm{BOR}_{1}$ antenna has only first-order variation in $\varphi$ and can be expressed as

$$
G_{y}(\theta, \varphi)=G_{E}(\theta) \sin \varphi \hat{\theta}+G_{H}(\theta) \cos \theta \hat{\varphi}
$$

for the y-polarized case. The $\mathrm{BOR}_{1}$ efficiency accounts for the power radiated in higher order $\varphi$-variations in the pattern, which cannot contribute to the gain of a symmetrical reflector antenna and thus represents a power loss. The spillover efficiency $e_{\mathrm{sp}}$ is the fraction of the radiated power that actually hits the reflector. The power lost in the cross-polar part of the field is accounted for by the polarization efficiency $e_{\mathrm{pol}}$. The illumination efficiency $e_{\text {ill }}$ is a measure of the loss that arises from the actual tapered illumination of the reflector, and finally the phase efficiency $e_{\phi}$ accounts for loss due to phase errors in the copolar aperture field. This efficiency is the only efficiency that depends on the
TABLE I

Cosmic Sky NoIse Toward the Galatic Pole

\begin{tabular}{cccc}
\hline \hline Frequency [GHz] & $\mathrm{T}_{\mathrm{S}}[\mathrm{K}]$ & Frequency [GHz] & $\mathrm{T}_{\mathrm{S}}[\mathrm{K}]$ \\
\hline 0.01 & 400000 & 0.60 & 9.0 \\
0.02 & 60000 & 0.80 & 6.0 \\
0.03 & 20000 & 1.00 & 4.5 \\
0.06 & 2800 & 3.00 & 2.8 \\
0.08 & 1000 & 6.00 & 2.8 \\
0.10 & 245 & 8.00 & 2.7 \\
0.30 & 30 & 10.00 & 2.7 \\
\hline
\end{tabular}

location of the feed relative to the focal point of the reflector. The feed location that maximizes the phase efficiency defines the phase center, see ([24, Sec. 2.3]). When calculating the phase efficiency below we have used the center of the ground plane as phase reference point.

\section{B. Figure of Merit}

For radio telescopes, such as SKA, the figure of merit for the overall system performance is effective area over the total system noise temperature $T$ given in $m^{2} / K$, i.e.,

$$
A / T=\frac{e_{\mathrm{rad}} e_{\mathrm{ap}} A_{\text {geom }}}{T}
$$

where $A_{\text {geom }}=\pi D^{2} / 4$ is the geometrical aperture area of the reflector, and $T$ is the system noise temperature. For a receiver with an isolator at temperature, $T_{\text {iso }}$ at its input the system temperature can be expressed as

$T=T_{R}+e_{\mathrm{rad}} p_{g} T_{a}+e_{\mathrm{rad}}\left(1-p_{g}\right) T_{\mathrm{sky}}+T_{\mathrm{ISO}} \frac{\left(1-e_{\mathrm{rad}}\right)}{e_{\mathrm{rad}}}$

with $T_{R}=15 \mathrm{~K}$ a typical receiver noise temperature, $T_{a}=$ $290 \mathrm{~K}$ the ambient temperature causing ground radiation, and $T_{\text {sky }}$ the cosmic sky noise. The feed will be located inside a Dewar cooled to $15 \mathrm{~K}$ which is assumed in (6). For the same reason the ohmic loss in the feed is neglected in this paper, so $e_{\text {rad }}=1-|r|^{2} \cdot p_{g}$ is the fraction of the radiated power in the radiation pattern of the feed which illuminates the ground. For prime focus case and when we assume that the antenna is pointed vertically, it can be calculated as

$$
p_{g}=p_{\text {gr }}+t_{\text {mesh }} p_{\text {mesh }}
$$

where $p_{\mathrm{gr}}$ is the relative power between $\theta=67$ and $\theta=90^{\circ}$, and $p_{\text {mesh }}$ is the relative power between $53^{\circ}$ and $67^{\circ}$, hitting the mesh reflector. $t_{\text {mesh }}$ is the transmission coefficient of the mesh. We will assume that the mesh is the same as the mesh in [27, Section IV], only scaled down in frequency by a factor 10 . The transmission coefficient is then about $1 \%$ at 500 and increases to $5 \%$ at $1.5 \mathrm{GHz}$, but we have assumed that it remains constant at $5 \%$ over the entire 0.15 - to $1.5-\mathrm{GHz}$ band. The cosmic sky noise toward the galactic center is found from [28, Fig. 2]. We approximate this by the values in Table I, with linear interpolation in $\log (\mathrm{T})$ versus $\log (\mathrm{f})$ between the values. For other pointing directions the spillover hitting the ground is modified, see, e.g., the formulas in [5], but we will not include such analysis here. 


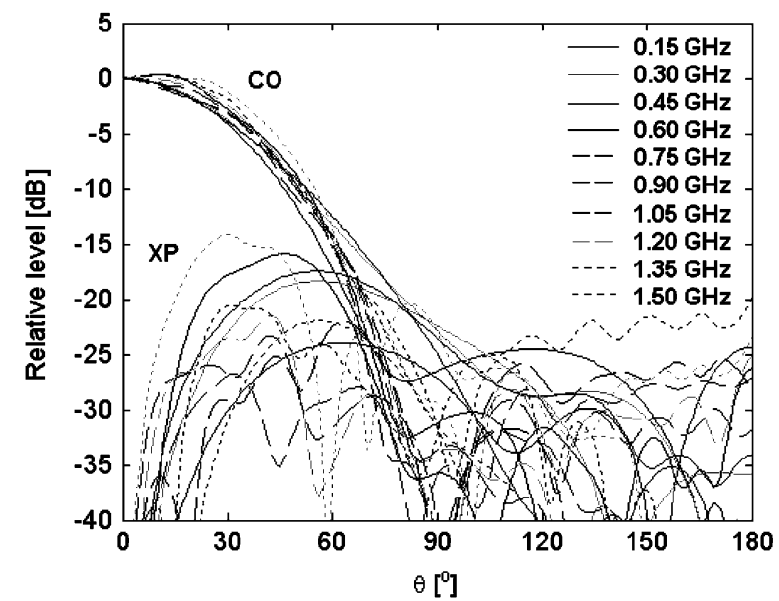

Fig. 3. Computed co- and cross-polar far-field patterns in the $45^{\circ}$ plane of the antenna in Fig. 2. BOR ${ }_{1}$-components only.

\section{NUMERICAL DESIGN}

We started by considering a simple feed consisting of two parallel halfwave dipoles separated by a half wavelength and located above a ground plane. This is as explained in the introduction an old feed which gives a pattern with equal E- and $\mathrm{H}$-planes and with phase center in the ground plane [3]. Our idea was then to replace each single dipole by log-periodic series-fed dipoles, with a logarithmically varying dipole length, spacing and height above the ground plane, see Fig. 2. The initial studies were made with wire dipoles, and we soon found out that the dipoles needed to be folded and mounted together in such a way that the feed gap of each folded dipole is connected to a gap in the normally connected folded part of the previous dipole. The studies were later concentrated more on strip models of the dipoles to enable printing on a normal dielectric circuit board.

To calculate impedance characteristics and radiation patterns of the feed we have mainly used the method of moments (MoM) as implemented in the commercial code WIPL-D [29], but we also used the in-house MoM code described in [30] for some initial studies and validation.

Here we will present numerical results obtained for the feed in Fig. 2 operating from 0.15 to $1.5 \mathrm{GHz}$, thereby covering a decade bandwidth. Note that we believe that, in principle, there exists no theoretical limit to the bandwidth of the log-periodic Eleven antenna, but our objective in the SKA application is to design decade bandwidth feeds. In practice the bandwidth is naturally limited by the size of the feed at low frequencies and by the diameter of the feeding cables at higher frequencies. Fig. 2 shows the most important longest dimensions of the feed. These sizes are also given in terms of the wavelength at $100 \mathrm{MHz}$. All the dimensions of the dipoles such as length $\mathrm{L}$, separation $\mathrm{D}$, height above ground plane $\mathrm{h}$, and strip width, are scaled with frequency to provide the log-periodic shape. The scaling factor $\mathrm{k}$ is 1.1161 . The feed is excited with a delta voltage source between the two strips of the split transmission line originating in the center, and the dielectric support is neglected. Furthermore the initial simulations were done for one linear polarization to reduce computational effort. The finite ground plane is included in the analysis. Fig. 3 shows typical far-field patterns in the $45^{\circ}$ plane between 0.15 and $1.5 \mathrm{GHz}$ similar to the patterns shown

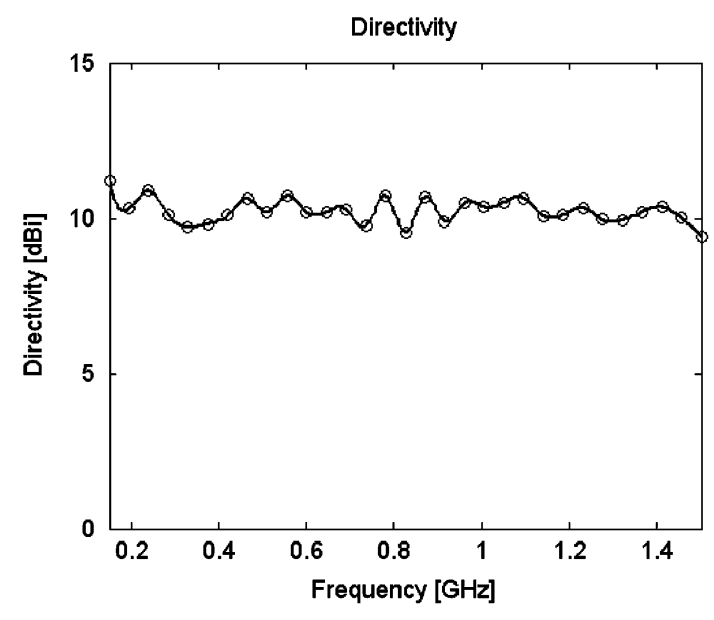

Fig. 4. Computed directivity of the antenna in Fig. 2.

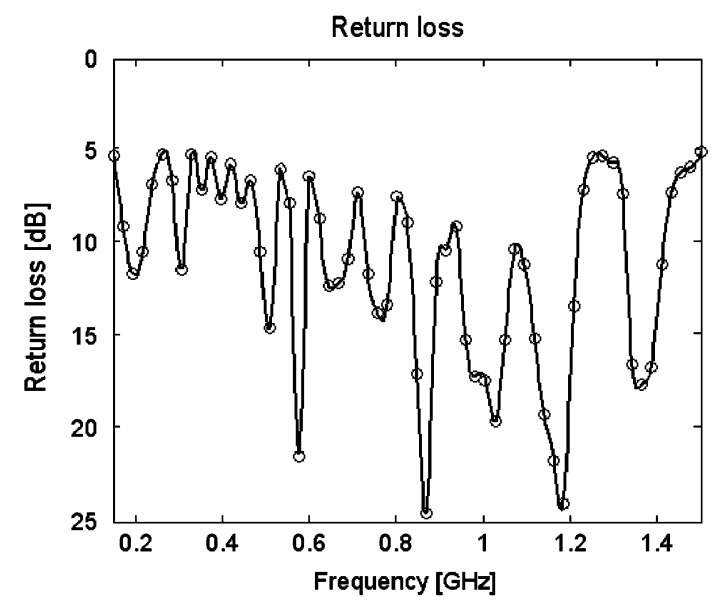

Fig. 5. Computed return loss of the antenna in Fig. 2.

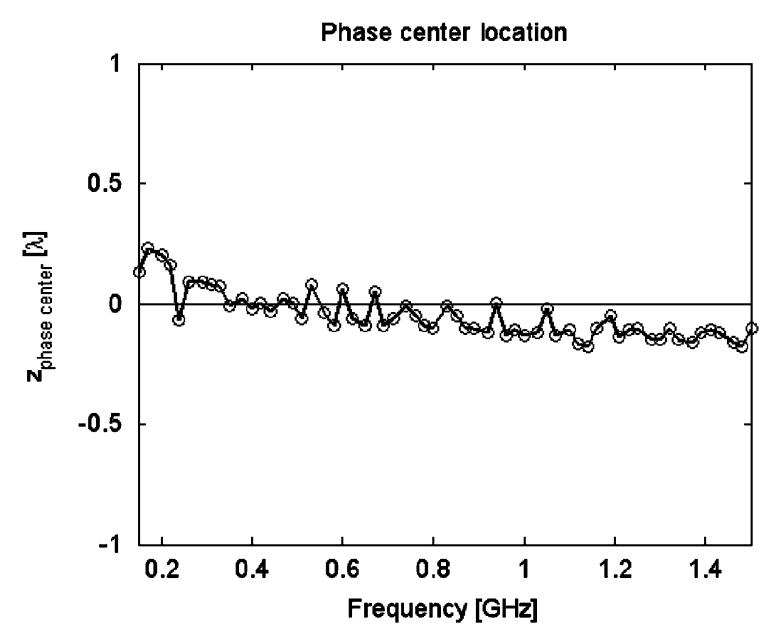

Fig. 6. Computed phase center location of the antenna in Fig. 2.

in [22] for an eleven antenna covering 1 to $12 \mathrm{GHz}$. Fig. 4 shows the directivity of the feed as a function of frequency. In Fig. 5 we see the return loss of the feed at the feed point. This constitutes the main drawback of the feed. The return loss is not better than $5.0 \mathrm{~dB}$ at the worst frequencies, but we are confident it can be improved. One of the major advantages of the feed is shown in Fig. 6 which presents the phase center location as a function 


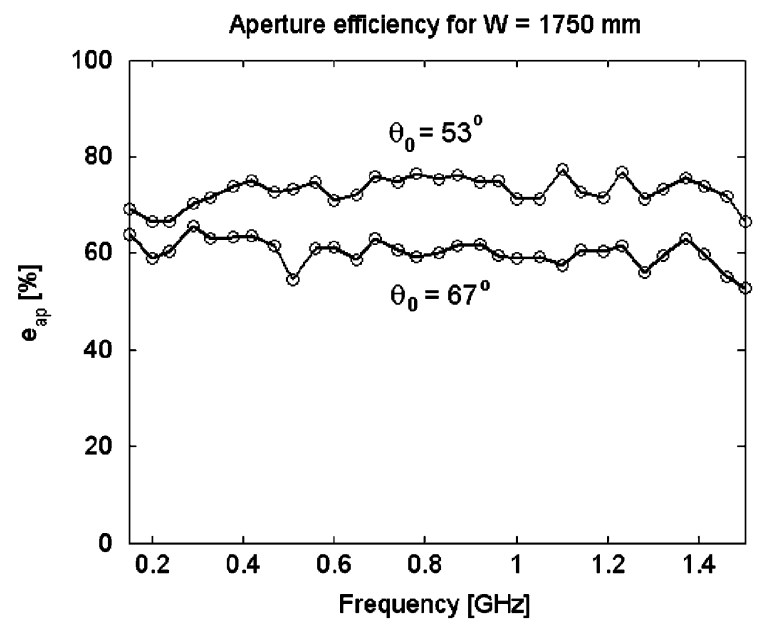

Fig. 7. Computed aperture efficiency when the antenna in Fig. 2 is used to feed a reflector with subtended angle of $53^{\circ}$ and $67^{\circ}$.

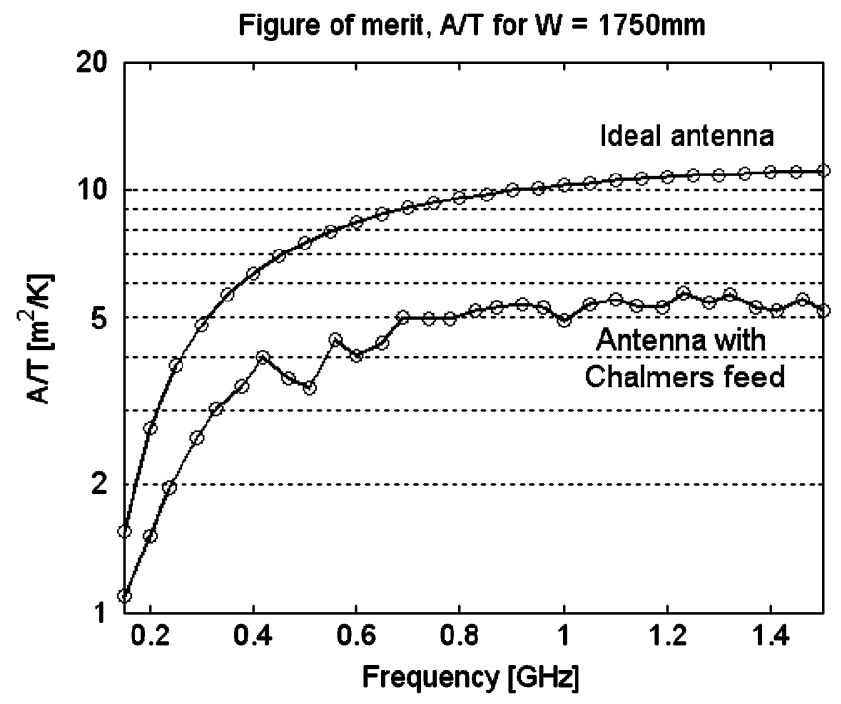

Fig. 8. Computed A/T of the reflector in Fig. 1 fed by the feed in Fig. 2. The ground plane size is $1750 \mathrm{~mm}$ or 0.88 wavelengths at $150 \mathrm{MHz}$, the lowest frequency of operation.

of frequency. We see that the phase center deviates by less than 0.2 wavelengths from the ground plane at $z=0$. Figs. 7 and 8 show the aperture efficiency and figure of merit in the reflector system, respectively. Fig. 7 shows that the subtended half angle influences the aperture efficiency quite significantly. In [22], the effect of the subtended half angle on the aperture efficiency was, therefore, studied and the optimum angle was shown to be close to $50^{\circ}$. A rather large range of subtended angles, from $40^{\circ}$ to $60^{\circ}$, can however be used and still remain very close to the maximum efficiency. The strong reduction of $\mathrm{A} / \mathrm{T}$ at low frequencies is not an effect of the feed but an effect of an increase in sky noise, see Table I. The A/T in Fig. 8 is compared to the theoretical maximum that would be achieved with an antenna with unity aperture efficiency and no spillover over the entire band.

Table II shows a summary of the different efficiencies of the feed. Worth noting here is that the phase efficiency is very high over the entire band, which was one of the main goals and indicates that the phase center location is fixed to the ground plane for all frequencies.
TABLE II

SUMMARY OF EFFICIENCIES OVER THE 0.15- TO 1.5-GHz BAND FOR THE FEED IN FIG. 3 FOR A SUbTENDED HALF ANGLE OF 53 DEG

\begin{tabular}{|c|c|c|c|c|}
\hline $\begin{array}{l}\text { Cause of } \\
\text { efficiency }\end{array}$ & Best $[\mathrm{dB}]$ & Worst $[\mathrm{dB}]$ & Best $[\%]$ & Worst $[\%]$ \\
\hline Spill-over, $\mathrm{e}_{\mathrm{sp}}$ & -0.26 & -0.69 & 94 & 85 \\
\hline Illumination, $\mathrm{e}_{\mathrm{ill}}$ & -0.41 & -0.74 & 91 & 84 \\
\hline Polarization, $\mathrm{e}_{\mathrm{pol}}$ & -0.01 & -0.15 & 100 & 97 \\
\hline Phase, $\mathrm{e}_{\phi}$ & -0.00 & -0.17 & 100 & 96 \\
\hline Aperture, $\mathbf{e}_{\mathrm{ap}}$ & -1.12 & -1.78 & 77 & 66 \\
\hline Reflections, $\mathrm{e}_{\text {refl }}$ & -0.02 & -1.44 & 100 & 72 \\
\hline Total, $\mathbf{e}_{\text {tot }}$ & -2.05 & -3.31 & 62 & 47 \\
\hline
\end{tabular}

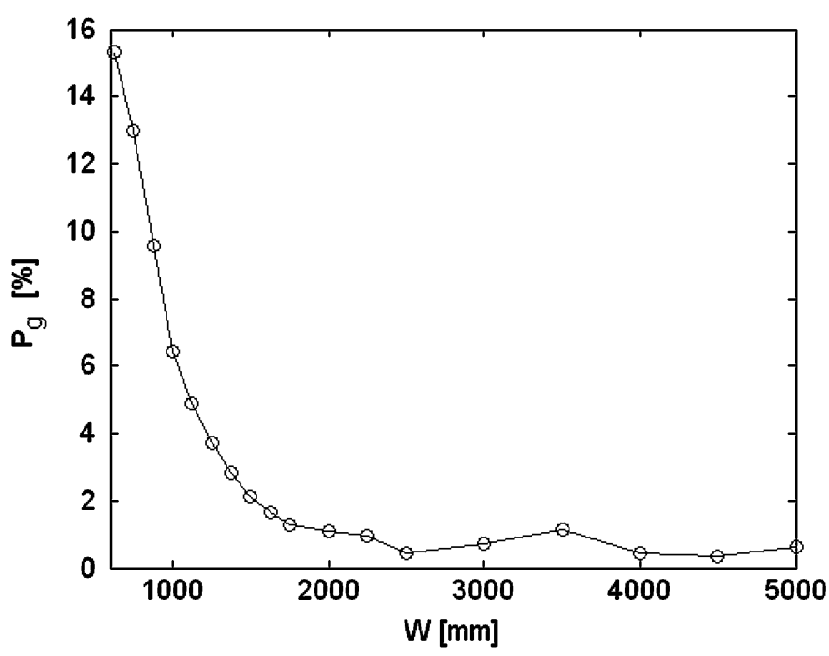

Fig. 9. Relative spillover at $150 \mathrm{MHz}$ hitting ground as defined by (7) versus ground plane size, computed for of the antenna in Fig. 2 when it is feeding the reflector in Fig. 1.

The far-out sidelobes and spillover can be reduced by increasing the size of the ground plane. This can of course only be done if practical size constraints allow it and if the increase in blockage is acceptable. Fig. 9 shows the percentage of the total radiated power which misses the reflector and hits the ground when the reflector is pointed normal to the ground. We refer to this quantity as relative power hitting the ground and its definition is stated in (7). We see that the spillover decreases significantly when the ground plane size increases, up to a ground plane size of around $1750 \mathrm{~mm}$ after which there is very little reduction of the spillover. This motivates the choice of ground plane size in the previous paragraph. This improvement in spillover should be traded off against the increased blockage loss, which we have not considered yet.

\section{Lab Model and Measurement}

The first experimental feed was linearly polarized and designed to cover 1 to $6 \mathrm{GHz}$. It is shown in Fig. 10. Fig. 11 shows a photograph of the central part. We see the soldering points where the inner conductor of the two feeding coaxial cables are connected to the strip lines. These cables come up through the ground plane via a centrally located hole and the shields of the cables are soldered to the ground plane. Each of the two parallel strip lines are fed by separate coaxial cable. Each coaxial cable 


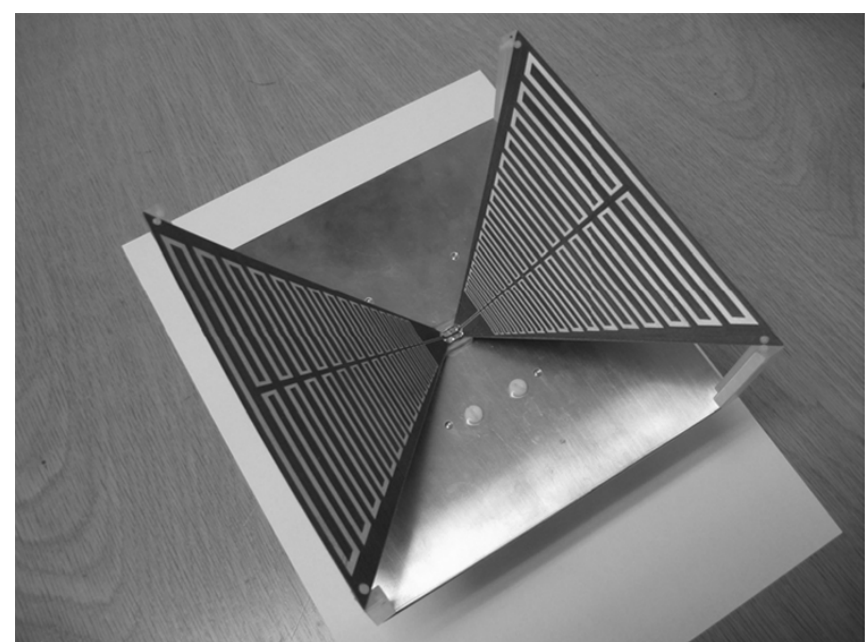

Fig. 10. Photograph of the 1- to 6-GHz lab model [23].

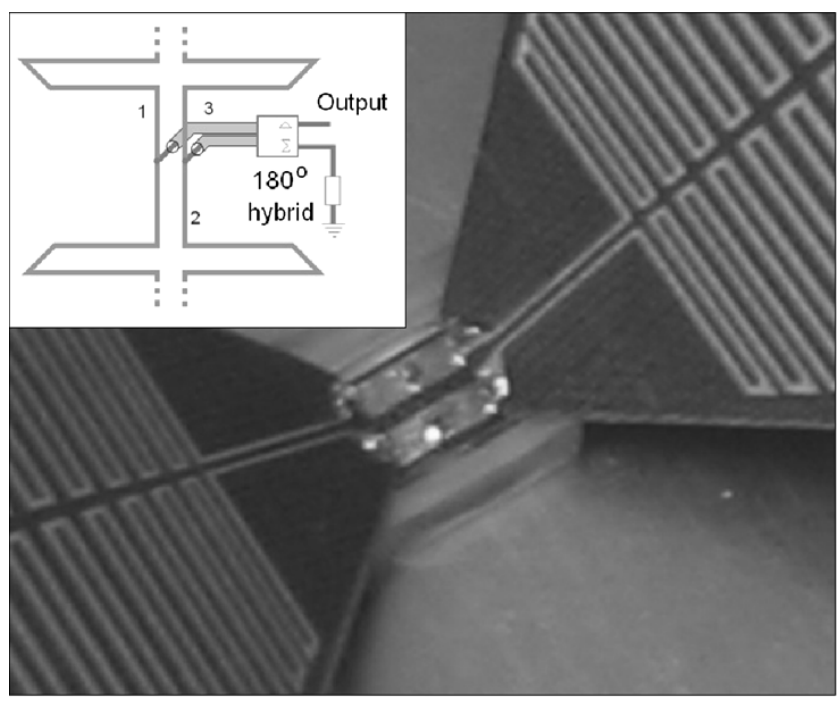

Fig. 11. Photograph of the central part of the 1- to 6-GHz lab model [23]. The inset shows a sketch of the feeding structure. Each of the two transmission lines, 1 and 2 , are fed by a separate coaxial line, 3 .

is, on the back side of the ground plane, connected to a commercial $180^{\circ}$ hybrid. The inset in the photograph shows a more illustrative sketch of the exciting structure.

The main features of the theoretical far-field patterns are well reproduced in the measurements. To illustrate this we plot two of the most important pattern parameters in Fig. 12: The 10-dB beam width and the highest cross-polar sidelobe. Both values are on average in agreement with simulations using the same model as described in Section III. The differences we see at certain frequencies probably arise from measurement inaccuracies, in particular when it comes to the cross polar levels, and from the fact that the excitation is not modeled with sufficient detail in the computations.

In Fig. 13, we compare typical measured and calculated farfield patterns at four frequencies. We see that the agreement is fairly good. The computed and measured reflection efficiencies are shown in Fig. 14. The effect of the feed cables and the $180^{\circ}$ hybrid have been removed by appropriate calibration. We see

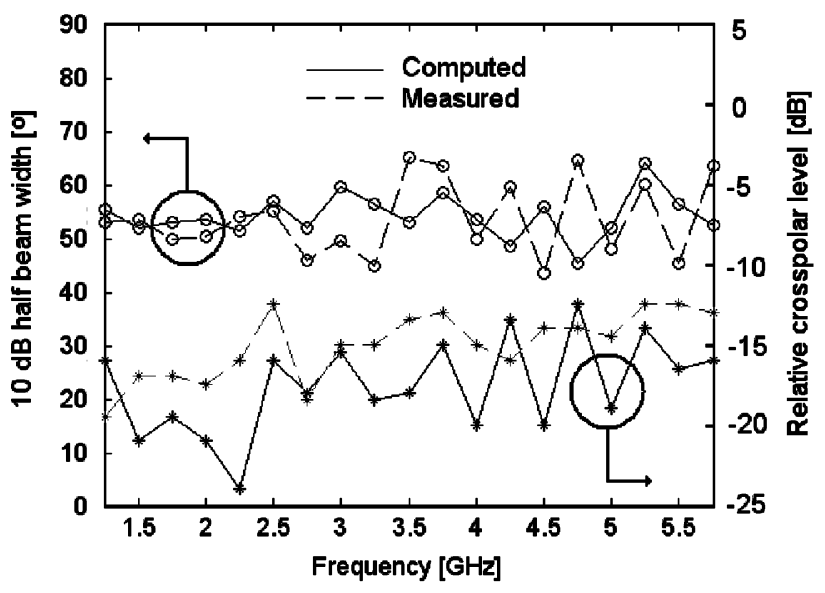

Fig. 12. Measured and computed $10 \mathrm{~dB}$ half beam width and highest cross-polar side lobe level in the $45^{\circ}$ plane for the 1 to $6 \mathrm{GHz}$ lab model.

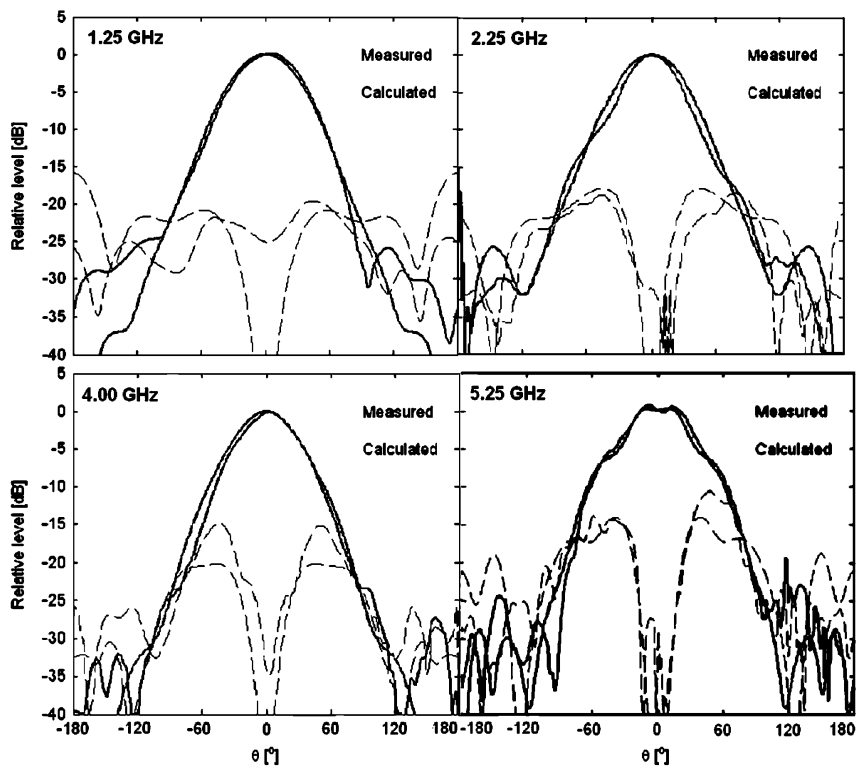

Fig. 13. Measured and computed co- and cross-polar far-field patterns in the $45^{\circ}$ plane at four frequencies.

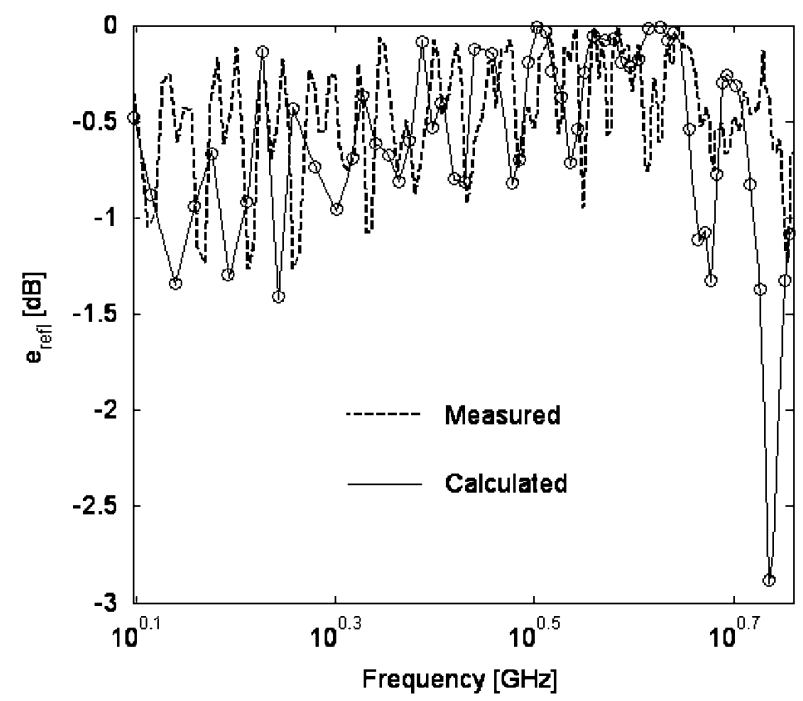

Fig. 14. Computed and measured reflection efficiency for the 1 to $6 \mathrm{GHz}$ lab model plotted against logarithmic scale in frequency. 


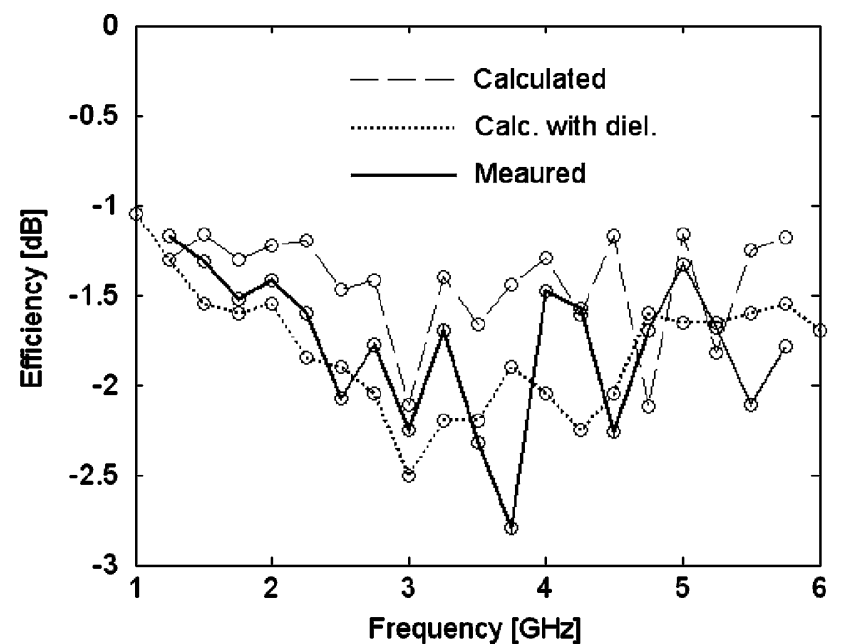

Fig. 15. Measured and computed product of polarization-illumination- and spillover efficiency for a subtended angle of $53^{\circ}$.

that the lowest level of the reflection efficiency is well predicted, but the more detailed behavior of the reflection efficiency is only approximately predicted by the simulations. We note that the measured reflections at the input cause up to $1.3 \mathrm{~dB}$ loss. The prediction of the pattern and the reflection efficiency shows that our numerical feed model provides useful and fairly accurate results.

We saw in the previous paragraph that the measured and calculated far-field patterns are similar. To quantify their similarity we have computed the efficiency of the feed in the SKA reflector, by using both the computed and simulated feed far-field patterns. Fig. 15 shows the product of the polarization-, illumination-, and spillover efficiencies. This product corresponds to the aperture efficiency, since the phase- and $\mathrm{BOR}_{1}$-efficiencies are close to $0 \mathrm{~dB}$. In this figure, we have also included results of simulations when the dielectric support boards are included. Then, the simulations predict the aperture efficiency more accurately. The reasons for the discrepancies are the simplified excitation model, an imbalanced feeding due to imperfections of the hybrid, and measurement inaccuracies. However, the aperture efficiency is overall well predicted.

\section{CONCLUSION}

A novel UWB feed called the Eleven antenna has been designed and manufactured. The feed provides nearly constant beam width and directivity over at least a decade bandwidth. It is also shown to give constant aperture efficiency of around $66 \%$ or better in the reflector system suggested for US-SKA over the same decade bandwidth and an A/T which is around $55 \%$ of that of an ideal antenna with $100 \%$ aperture efficiency and no spillover. It has been shown that the spillover efficiency can be controlled by changing the size of the ground plane. We have also shown that the feed can be used in dual linear polarization with little degradation of performance.

There are many things that still can be done to improve this feed. One of the key issues is to improve impedance matching and ongoing work shows promising results. Another issue is to study the losses introduced by the dielectric substrate and the finite conductivity of the metal parts.

\section{REFERENCES}

[1] M. I. Skolnik, Introduction to Radar Systems. New York: McGrawHill, 1962.

[2] S. Silver, Microwave Antenna Theory and Design. New York: McGraw-Hill, 1949.

[3] S. Christiansen and Högbom, Radiotelescopes. Cambridge, U.K.: Cambridge Univ. Press, 1969.

[4] P.-S. Kildal and S. A. Skyttemyr, "Dipole-disk antenna with beam-forming ring," IEEE Trans. Antennas Propag., pp. 529-534, Jul. 1982.

[5] P.-S. Kildal, S. A. Skyttemyr, and A. A. Kishk, "G/T maximization of a paraboidal reflector fed by a dipole-disk antenna with ring by using the multple-reflection approach and the moment method," IEEE Trans. Antennas Propag., vol. 45, no. 7, pp. 1130-1139, Jul. 1997.

[6] B. Thomas, G. James, and K. Greene, "Design of wide-band corrugated conical horns for Cassegrain antennas," IEEE Trans. Antennas Propag., vol. 34, no. 6, pp. 750-757, Jun. 1986.

[7] B. M. Thomas, K. J. Greene, and G. L. James, "A wide-band primefocus horn for low-noise receiver applications," IEEE Trans. Antennas Propag., vol. 38, no. 11, pp. 1898-1900, Nov. 1990.

[8] E. Lier, "A dielectric hybrid mode antenna feed: A simple alternative to the corrugated horn," IEEE Trans. Antennas Propag., vol. 34, no. 1, pp. 21-29, Jan. 1986.

[9] P. R. Clark and G. L. James, "Ultra-wideband hybrid-mode feeds," Electron. Lett., vol. 31, no. 23, pp. 1968-1969, Nov. 9, 1995.

[10] P. J. B. Clarricoats, A. D. Olver, and B. Ok, "Broadband low-crosspolarization horn," Electron. Lett., vol. 30, no. 25, pp. 2085-2086, Dec. 8, 1994.

[11] J. Shimizu, "Octave-bandwith feed horn for paraboloid," IEEE Trans. Antennas Propag., vol. 9, no. 2, Mar. 1961.

[12] C. Bruns, P. Leuchtmann, and R. Vahldieck, "Analysis and simulation of a 1-18-GHz broadband double-ridged horn antenna," IEEE Trans. Electromagn. Compat., vol. 45, no. 1, pp. 55-60, Feb. 2003.

[13] Z. Ying, A. A. Kishk, and P.-S. Kildal, "Broadband compact horn feed for prime-focus reflectors," Electron. Lett., vol. 31, no. 14, pp. 1114-1115, Jul. 1995

[14] A. Clavin, "A new antenna feed having equal E -and H-plane patterns," Trans. IRE Professional Group on Antennas Propag., vol. 2, no. 3, pp. 113-119, Jul. 1954.

[15] J. Yang and P.-S. Kildal, "Calculation of ring-shaped phase centers of feeds for ring-focus paraboloids," IEEE Trans. Antennas Propag., vol. 48, no. 4, pp. 524-528, Apr. 2000.

[16] C. L. Carilli and S. Rawlings, Science with the Square Kilometer Array: Motivation, Key Science Projects, Standards and Assumptions, Sep. 2004.

[17] D. E. Isbell, "Log periodic dipole arrays," IRE Trans. Antennas Propag., vol. 8, no. 3, pp. 260-270, May 1960.

[18] W. A. Imbriale, "Applications of the method of moments to thin-wire elements and arrays," in Numerical and Asymptotic Techniques in Electromagnetics, R. Mittra, Ed. New York: Springer-Verlag, 1975, ch. 2

[19] R. Dybdal, "Defocusing loss for a log periodic-fed reflector," IEEE Trans. Antennas Propag., vol. 33, no. 7, pp. 809-812, Jul. 1985.

[20] G. Engargiola, "Non-planar log-periodic antenna feed for integration with cryogenic microwave amplifier," in IEEE Antennas and Propagation Soc. Int. Symp. UNSC/URSI National Radio Science Meetings, 2002, pp. 140-143.

[21] D. Ericsson, P.-S. Kildal, and S. Weinreb, "Study of efficiencies and phase centers of broadband log-periodic feeds for large offset dual-reflector antennas using formulas for bodies of revolution (BOR1 extraction)," in Proc. IEEE AP-S Int. Symp. , Columbus, OH, Jun. 2003.

[22] R. Olsson, P.-S. Kildal, and S. Weinreb, "A novel low-profile log-periodic ultra wideband feed for the dual-reflector antenna of US-SKA," in Proc. IEEE AP-S Int. Symp., Monterey, CA, Jun. 2004.

[23] P.-S. Kildal, "Broadband Multi-Dipole Antenna with FrequencyIndependent Radiation Characteristics," Patent Application PCT/ SE2004/001 178.

[24] — Foundations of Antennas—A Unified Approach. Lund, Sweden: Studentlitteratur, 2000.

[25] — "Factorization of the feed efficiency of paraboloids and cassegrain antennas," IEEE Trans. Antennas Propag., vol. 33, no. 8, pp. 903-908, Aug. 1985.

[26] P. Kildal and Z. Sipus, "Classification of rotationally symmetric antennas as types BOR0 and BOR1," IEEE Antennas Propag. Mag., vol. 37, no. 6, p. 114, Dec. 1995.

[27] W. A. Imbraile, V. Galindo-Israel, and Y. Rahmat-Samii, "On the reflectivity of complex mesh surfaces," IEEE Trans. Antennas Propag., vol. 39, no. 9, pp. $1352-1365$, Sep. 1991 
[28] W. van Cappellen, , LOFAR-ASTRON-ADD-009 Rep., Oct. 2002.

[29] WIPL-D Pro. v5.1 3D Electromagnetic Solver Professional Edition User's Manual, 2004. WIPL-D Ltd..

[30] J. Carlsson and P.-S. Kildal, "Physical asymptotic model for a conducting patch and how this enables the inclusion of dielectric substrate in a free-space moment method code," in Inst. Electr. Eng. Proc. Microwave, Antennas and Propagation, vol. 151, Aug. 2004, pp. 338-344.

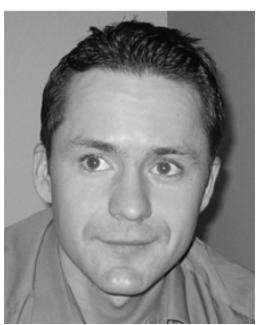

Rikard Olsson (M'04) was born in Umea, Sweden, in 1979. He received the Masters degree in engineering physics from Chalmers University of Technology, Goteborg, Sweden, in 2003.

$\mathrm{He}$ is currently pursuing the Ph.D. degree at Chalmers University of Technology.

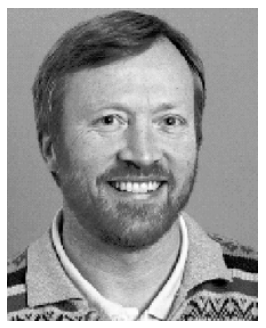

Per-Simon Kildal (M'82-SM'84-F'95) was born in Molde, Norway, on July 4, 1951. He received the M.S.E.E., Ph.D., and Doctor Technicae degrees from the Norwegian Institute of Technology (NTH), Trondheim, Norway, in 1976, 1982, and 1990, respectively.

From 1979 to 1989 , he was with the ELAB and SINTEF, research institutes in Trondheim, Norway. Since 1989, he has been a Professor at Chalmers University of Technology, Gothenburg, Sweden, where he has educated 15 antenna doctors. He has done the electrical design and analysis of two very large antennas. The first was the $120-\mathrm{m}$ long and 40-m wide cylindrical parabolic reflector antenna of the European Incoherent Scatter Scientific Association (EISCAT), located in North Norway. The second was the Gregorian dual-reflector feed of the 300-m diameter radio telescope in Arecibo, on a contract for Cornell University. He is presently involved in the design of feeds for the U.S. proposal of the Square Kilometer Array (SKA). He holds many granted patents and patents pending, and based on these he has founded two companies: COMHAT AB (www.comhat.se) and Bluetest AB (www.bluetest.se). He has authored or coauthored more than 190 papers in IEEE TRANSACTIONS or IEE journals and conferences, concerning antenna theory, analysis, design and measurement. He gives short courses and organizes special sessions at conferences, and he has given invited lectures in plenary sessions at several conferences. His textbook Foundations of Antennas-A Unified Approach (Lund, Sweden: Studentlitteratur, 2000) (www.studentlitteratur.se/antennas) has been well received.

Prof. Kildal received an award from ELAB in 1984. He received the R. W. P. King Award in 1984 and the S.A. Schelkunoff Transactions Prize Paper Award in 1990 from the IEEE TRANSACTIONS ON ANTENNAS AND PROPAGATION. From 1995 to 1998, he served as an Associate Editor of the IEEE TRANSACTIONS ON ANTENNAS AND PROPAGATION and was Co-Guest Editor of the 2005 IEEE Transactions on ANTENNAS AND PROPAGation Special Issue on Artificial Magnetic Conductors, Soft/Hard Surfaces, and Other Complex Surfaces. He has made many contributions to the IEEE Antennas and Propagation Society including being an elected member of the Administration Committee during 1995-1997 and distinguished lecturer from 1991 to 1994
Sander Weinreb (LF'02) received the B.S.E.E. and Ph.D. degrees from the Massachusetts Institute of Technology (MIT), Cambridge, in 1958 and 1963 , respectively.

He is presently a Principal Scientist at the Jet Propulsion Laboratory (JPL) and a Faculty Associate at the California Institute of Technology (Caltech). His present area of interest is large microwave arrays for space communications and radio astronomy. He is active in research and development of very low-noise microwave and millimeter wave integrated circuits (MMICs). Prior to joining JPL, he was a Research Professor with the Department of Physics and Astronomy at the University of Massachusetts (1996-1999). He was Principal Scientist and Leader of the Millimeter-Wave Design and Test Group at Martin Marietta Laboratories where he led the design of millimeter-wave MMICs and prototype radar and radiometer systems (1989-1996). Prior to this, he was Head of the Electronics Division (1965-1985) and Assistant Director (1985-1988) at National Radio Astronomy Observatory, where was responsible for the design, construction, operation, and maintenance of radio astronomy receivers at the Green Bank, WV, and Kitt Peak, AZ observatories. At NRAO, he led the group which was responsible for the design of the electronics system for the Very Large Array. He is the author of over 120 publications in the areas of digital correlation techniques, radio astronomy observations, array receivers, and low-noise amplifiers. 